Hannah Arendt, La Nature du totalitarisme. Trad. de l'anglais et préf. par Michelle-Irène B. de Launay. Paris, Payot, 1990. 14 × 22,5, 183 p. (« Bibliothèque philosophique Payot »).

Sous le titre La Nature du totalitarisme, l'ouvrage de $\mathrm{H}$. Arendt qui nous est offert présente trois articles. Deux relatifs au statut de la compréhension du phénomène totalitaire et un troisième renvoyant au rapport entre religion et politique. Véritables mises au point, ces articles permettent de lever deux ambiguïtés. La première concernant la compréhension du phénomène totalitaire prisonnier de l'alternative, qui a tendance à en faire un accident quand elle n'en fait pas un effet nécessaire de l'histoire. La seconde concernant la définition du phénomène totalitaire comme phénomène religieux. $\mathrm{H}$. Arendt le précise avec netteté. Le fait de se demander si le phénomène totalitaire est un accident ou un effet nécessaire est pervers, car cette façon de vouloir trouver ainsi une explication totale de l'histoire ne conduit qu'à nous faire manquer l'événement. Aussi nous propose-t-elle de renverser la démarche et de retourner à l'événement lui-même, en procédant comme Montesquieu, par l'analyse des structures et des mobiles qui meuvent les systèmes politiques. En l'occurrence, s'agissant du totalitarisme, celui-ci a été un régime inédit fondé sur la crainte et aboutissant à "l'esseulement " de l'humanité.

La même chose vaut quant au rapport religion-politique. Se demander si le totalitarisme a été ou non une religion est une autre façon d'en masquer la nature et de ne pas voir où se situe sa véritable portée religieuse, à savoir la perte de la crainte de l'enfer.

En fait, à travers deux angles différents, $H$. Arendt fait preuve de la même vigueur méthodologique et du même souci de poser les bonnes questions, nous donnant ainsi les moyens de voir en quoi quelque chose comme la destruction totalitaire du sens commun fait événement et situe le totalitarisme bien au-delà d'un simple accident ou d'un effet logique de l'histoire.

Bertrand Vergely.

\title{
CULTURE POLITIQUE ET ENSEIGNEMENT
}

Anthony Grafion, Forgers and Critics. Creativity and Duplicity in Western Scholarship. Princeton, NJ, Princeton University Press, 1990. 14,5 × 22,3, 157 p., index.

Ce court volume développe une "Public Lecture " donnée par l'Auteur à l'université de Princeton. Sur un problème précis (celui de la relation entre le faussaire 
et le critique), A. Grafton déploie brillamment quelques-unes des vues grâce auxquelles son œuvre marquera l'histoire de l'érudition occidentale. Vues qui ne sont pas sans analogie avec celles qui ont prévalu en histoire des sciences depuis un demi-siècle, des deux côtés de l'Atlantique.

La thèse principale soutenue par Grafton, c'est qu'on ne peut opposer le critique au faussaire comme la vertu au vice ou le porteur de lumière à l'ange des ténèbres. L'un et l'autre ont partie liée. Grafton, pour mieux faire voir le phénomène, promène sa caméra sur l'histoire de l'érudition occidentale en changeant l'angle des prises de vues.

1) Le faussaire et le critique ne peuvent être séparés par une nette coupure temporelle, qui renverrait le premier à la nuit de l'Antiquité et du Moyen Âge, et placerait le second à un poste glorieux de pionnier, sur une large route qui, tout exposée au soleil de la raison, conduirait de la Réforme aux Lumières, puis à l'éclatante Wissenschaft du XIx ${ }^{e}$ siècle. Les Grecs, dès le Iv siècle avant JésusChrist, ont connu l'activité des critiques et celle des faussaires s'est poursuivie jusqu'à nos jours.

2) Les plus grands critiques ont pu se livrer à des imitations de l'antique pour servir leurs aspirations. Ainsi Érasme lui-même a-t-il ajouté le De duplici martyrio à son édition des cuvres de S. Cyprien en 1530 . Inversement, un faussaire aussi dénoncé qu'Annius de Viterbe a imposé à ses plus coriaces adversaires l'importance primordiale des " archives " comme source de connaissance historique et a entraîné les historiens de l'Antiquité à constituer des collections de fragmenta des œuvres perdues. Du débat entre faussaires et critiques, au $x \mathrm{x}^{\mathrm{e}}$ siècle, naît la méthode historique recueillie par les érudits de la fin du Xvir siècle.

3) Faussaire et critique utilisent les mêmes arguments, puisqu'il s'agit de part et d'autre d'arrimer un document à un temps et à un lieu, grâce à une excellente connaissance de la langue et des usages de la société où le texte a vu (ou aurait vu) le jour. Chacun à sa manière, le faussaire et le critique contribuent côte à côte à affiner les instruments de la connaissance du passé.

4) Le faussaire et le critique portent chacun la marque de leur temps. Mais pas en ce sens que l'un appartiendrait au temps de la rumeur et de l'à-peu-près, l'autre au temps de la vérité et de la précision. Cette appréciation est elle-même fille des Lumières ou du scientisme. L'impitoyable caméra de Grafton montre que l'activité du faussaire apprend toujours quelque chose de «vrai » : le cas le plus banal est celui des légendes d'origine forgées par une cité, une nation, un ordre religieux. Mais, plus subtilement, les contrefaçons littéraires peuvent exprimer la résistance idéologique d'un groupe à la culture dominante (par exemple, celle qu'opposent à l'hellénisation les cultures antérieures aux conquêtes d'Alexandre, par la rédation du Corpus hermeticum). Le critique, lui, porte la marque de son temps, dans la mesure où, pour décider qu'une expression ou un usage sont anachroniques, il faut avoir une idée du passé, elle-même tout entière relative à l'époque de l'observateur.

Bref, Anthony Grafton montre de façon convaincante que les rapports de la vérité et de l'erreur sont plus complexes que prévu. L'effort de méthode est relatif à la conception qu'une époque se forge de la vérité et l'histoire des erreurs est instructive : elle ne se résume pas à une chronique de l'humaine déraison. Cela dit, Grafton affirme en terminant qu'il n'entend pas niveler, au plan éthique, le faus- 
saire et le critique, mais que la lucidité dont se réclame le critique le renvoie directement à sa propre généalogie.

François LAPLANCHE.

Haut Moyen Âge : culture, éducation et société. Études offertes à Pierre Riché. Ed. Centre de recherche sur l'Antiquité tardive et le haut Moyen Âge de l'université Paris X-Nanterre, préf. Michel Sor. La Garenne-Colombes, Publidix/Ed. européennes Erasme, 1990. $16 \times 24,630$ p., ill., bibliogr., index.

L'ouvrage réunit plus de quarante contributions internationales dont la richesse atteste le rayonnement de l'œuvre du professeur Pierre Riché : sa bibliographie, établie par Charles Vulliez, comptait près de cent cinquante titres en avril 1990.

Les études rassemblées ici " en écho " à ces travaux soulignent le rôle essentiel du haut Moyen Âge dans la transmission de la culture antique, remodelée par le christianisme, aux siècles ultérieurs. Dans les limites de ce compte rendu, il n'est pas possible de détailler chacun de ces travaux. Contentons-nous d'illustrer par quelques exemples la diversité et la fécondité des méthodes d'approche mises ici en œuvre (philologie, sémiotique, hagiographie, numismatique, diplomatique, voire musicologie...).

Une première partie ("Antiquité tardive et occident barbare ") regroupe treize études. Celle qu'A. Chastagnol consacre, cum grano salis, au « Salaire des professeurs à Rome " prouve que le système des écoles publiques de l'Antiquité et le mode de rétribution des maitres en nature (l'annone, apparue au $\mathrm{rv}^{\varepsilon}$ siècle) ont perduré, du moins à Rome, jusqu'en plein vie siècle. Pour la Gaule, le dernier témoignage explicite date de 474 . Cependant, l'analyse systématique par M. Heinzelman ( "Studia sanctorum. Éducation, milieu d'instruction et valeurs éducatives dans l'hagiographie en Gaule jusqu'à la fin de l'époque mérovingienne ») d'un corpus de cent cinq Vitae confirme que les évêques ont assuré la continuité de l'enseignement public dans leurs " cités ". Bien entendu, l'enseignement religieux occupait une place prépondérante dans les programmes, mais les familles favorisées "recommandaient " leurs enfants à des personnalités aptes à garantir à ceux-ci des carrières ultérieures, tant laïques qu'ecclésiastiques. On constate ainsi de notables promotions sociales, comme dans le cas de saint Éloi dont J. Lafaurie prouve ( Le monnayage mérovingien : témoin de quatre siècles d'histoire ") qu'il a organisé la première dévaluation de la monnaie d'or. M. Reydellet (« Venance Fortunat et l'esthétique du style ") analyse finement la production littéraire de Fortunat, immigré italien promu évêque de Poitiers à la fin du vie siècle. Celui-ci parvient à capter les faveurs d'un public composé essentiellement de notables austrasiens férus d'asianisme, tout en se préoccupant de création poétique. Conscient de la « fracture culturelle ", il se montre l'initiateur de la poésie médiévale. J. Le Goff ("Le rire dans les règles monastiques du haut Moyen Âge ") marque une nouvelle étape dans sa réflexion sur le rire médiéval. Ces sources normatives font apparaître l'oscillation du monachisme entre deux conceptions chrétiennes du 
rire : l'une d'origine orientale, transmise à la chrétienté latine par Rufin qui prohibe le rire dans la mesure où le Christ n'a jamais ri ; l'autre, influencée par Aristote, selon laquelle le rire est le propre de l'homme. C'est la première qui s'impose, proposant au moine le modèle de l'homo lugens, comme l'atteste, au début du $\mathrm{x}^{e}$ siècle, le Codex Regularum de Benoît d'Aniane, synopse des règles anciennes destiné à démontrer la supériorité de celle de saint Benoit.

Les réformes incarnées par Benoît d'Aniane ne constituent qu'un des aspects de la « Renaissance " carolingienne qu'illustrent les onze contributions qui forment la seconde partie (« L'Europe carolingienne »). Dans le domaine de la liturgie, I. Reznikoff (" Le chant des Gaules sous les Carolingiens ") établit le dossier des textes carolingiens concernant le chant « grégorien » (752-850) et prouve que, si les liturgistes ont effectivement adopté l'ordo romain pour la célébration, la mélodie s'en est tenue à la tradition orale de la Gaule du Nord-Est et de l'Aquitaine. Plusieurs études montrent que l'hagiographie connaît un regain de vitalité au cours du $\mathrm{rx}^{\varrho}$ siècle. François Kerlouégan (« Landevennec à l'école de SaintSauveur de Redon? ") réplique ici à un article du père Marc Simon de Landevennec (M.S.H.A.B., 63, 1986) qui, beau joueur, supposait que les hagiographes de son monastère avaient pu être formés à Redon. Tout en admettant l'éventualité de contacts entre les deux grandes abbayes bretonnes, l'auteur insiste sur la supériorité intellectuelle de celle de Landevennec, tournée vers le monde insulaire. Doté d'une riche bibliothèque et d'un scriptorium fécond, ce monastère produit, en quelques décennies, diverses Vitae qui se distinguent par une latinité plus ambitieuse et moins conventionnelle que celle des Gestes des saints de Redon. Évidemment, ces clercs lettrés ne constituaient qu'une petite élite parmi une masse illettrée. Cependant, comme le remarque $\mathrm{M}$. Mostert ( $\mathrm{La}$ magie de l'écrit dans le haut Moyen Âge "), cette dernière n'avait pas, pour autant, une " culture d'oralité pure ». L'écrit revêtait, à ses yeux, un statut magique que les lettrés lui reconnaissaient aussi, d'ailleurs, ne serait-ce que pour conforter leur pouvoir : phylactères apparentés aux « lettres de délivrance "; fréquence du motif de la " lettre tombée du ciel $»$, autant de témoignages de cette magie de l'écrit dont l'auteur propose une typologie provisoire.

À ce propos, l'Historia Remensis Ecclesiae (IV, 48) de Flodoard rapporte comment un saint pèlerin irlandais, assassiné depuis longtemps, apparaît à un clerc pour lui dicter son nom et corriger les fautes d'orthographe commises par celuici! L'étude de ce document par M. Sot (" L'expérience visionnaire et sa fonction dans l'Histoire de l'Église de Reims de Flodoard ") illustre, parmi une dizaine d'autres communications (" Le $x^{e}$ siècle : un autre grand siècle "), le renversement des perspectives sur le $x^{e}$ siècle provoqué par les propres recherches du dédicataire de l'ouvrage. La " Renaissance " carolingienne est encore alors fort féconde. À partir d'un corpus de 29 récits de visions (14 appréhendées par des sources hagiographiques antérieures; 15 transmises par la tradition orale, non sans qu'il y ait interference entre les deux types de récits), M. Sot analyse la théologie de l'histoire qui sous-tend l'œuvre de Flodoard : les visions sont, pour celui-ci, des faits contrôlables par les sens qui fondent l'histoire du diocèse de Reims dans le sacré.

La dernière partie ("Mémoire du haut Moyen Âge ») regroupe six études qui insistent, à des titres divers, sur les continuités par lesquelles le haut Moyen Âge est resté une période de référence pour les siècles suivants. K. F. Werner ( $\mathrm{La}$ 
légende de saint Alexis : un document sur la religion de la haute noblesse vers l'an mil ») discerne, à l'origine de la chanson en vieux français (v. 1040), une légende orientale du haut Moyen Âge adaptée en latin au $x^{e}$ siècle. Le texte reflète la fascination de l'Occident latin pour la civilisation byzantine et s'il annonce, par certains traits, la Chanson de Roland, c'est qu'il s'adresse au même public noble et plus particulièrement à la haute aristocratie de l'entourage du roi Robert II. À titre d'hypothèse, l'auteur soupçonne des liens éventuels entre les aspects antimatrimoniaux de cette légende et les courants hérétiques qui touchent alors ces mêmes milieux. N. Y. Tonnerre (" Une terre celtique : le Cornwall à la lecture du Domesday Book ») compare la Comouaille insulaire du $\mathrm{xI}^{\mathrm{e}}$ siècle et la Bretagne centrale telle que la fait connaitre le Cartulaire de Redon. Les rapports sont plus intenses de part et d'autre de la Manche qu'entre Cornwall et Pays de Galles : il y a d'ailleurs encore intercommunication linguistique. A. Vauchez ( Le duc Charles de Blois († 1364) et le culte des saints rois bretons du haut Moyen Âge ") exploite plusieurs témoignages sur la dévotion du duc de Bretagne pour les saints " rois ", plus ou moins historiques, des origines bretonnes, notamment Judicaël et Salomon. Dans le cas de Charles de Blois qui se rattachait à la lignée capétienne, ce culte ne peut traduire une revendication de beata stirps. Par contre, on peut y voir la quête d'une légitimité par antériorité, dans le contexte de l'affirmation de l'identité bretonne perceptible aux $\mathrm{XIV}^{*} \mathrm{xv}^{6}$ siècles. De grandes familles revendiquent des ancêtres illustres : les Chastel prétendent remonter à saint Tanguy; les Rohan se réclament de saint Mériadec. Les franciscains observants optent, comme l'a montré $\mathrm{H}$. Martin, pour des établissements insulaires, par référence au monachisme celtique. Autant d'indices convergents de l'impact du lointain passé breton à la fin du Moyen Âge.

Ces quelques remarques ne donnent, bien entendu, qu'un aperçu partiel de l'intérêt de l'ouvrage, véritable panorama des travaux en cours dans le domaine de l'histoire de la culture et des mentalités dont le professeur P. Riché a montré l'importance. Qu'il soit permis, pour terminer, au signataire de ce compte rendu de témoigner modestement que ses anciens étudiants n'ont pas oublié la leçon.

Bernard MERdRIGnac.

The Transmission of Culture in Early Modern Europe. Eds Anthony Grafton, Ann Blarr. Philadelphie, University of Pennsylvania Press, 1990. $16 \times 23,5$, $336 \mathrm{p}$.

Les travaux américains sur l'histoire culturelle de l'Europe moderne s'amoncellent, presque toujours dûs aux « editors » du Journal of History of Ideas ou à leurs élèves. Leur caractéristique commune consiste à prendre une certaine distance par rapport à l'historiographie reçue, qui oppose trop facilement la modernité à la tradition, comme la lumière aux ténèbres, la vérité au mensonge, la rationalité à la fabulation. La thèse générale de "l'école américaine" (si je puis m'exprimer ainsi, par une simplification commode), consiste à soutenir que, quoi 
qu'en aient pensé les acteurs du changement, «le retour à l'Antiquité », qui sert d'appellation contrôlée à la culture de la Renaissance, n'est pas fondamentalement en rupture avec la culture médiévale (et, bien entendu, avec ce qu'elle charrie de culture antique). Il s'agit en fait d'un héritage. Mais ceci, qui a déjà été bien vu par Aby Warburg, demande encore à être précisé. Car le travail de réception ne se réduit pas, dans ce cas, à un nettoyage des " monumenta ", à une restauration de la culture antique (" déformée " par une transmission décadente, aussi bien en ce qui concerne les arts que les lettres). Il faut voir plutôt dans ce retour à l'antique une création originale où l'imagination prend autant de place que la raison et le rêve que la méthode. Les nouvelles formes de la culture savante s'originent toujours à la source d'un imaginaire collectif et, inversement, les formes non littéraires de la culture, loin d'exprimer la spontanéité "sauvage " d'un groupe, révèlent une organisation et une logique. Enfin, la pratique juridique d'une société donnée constitue un remarquable révélateur des interactions entre les intérêts économiques, les hiérarchies sociales et les mythes de cette société. Telles sont les principales affirmations posées par Anthony Grafton (professeur au département d'histoire de Princeton) dans une très belle introduction historiographique appelée à faire date.

Sa propre contribution au volume vise à provoquer une reconsidération du cas « Annius de Viterbe ». Les faux documents du dominicain italien, maître du Sacré Palais à la fin du $\mathrm{xv}^{e}$ siècle, sont fabriqués en fonction d'une stratégie culturelle précise : reconduire la mythologie grecque à ses sources orientales et donc à la Bible. Ce geste reprend le mouvement de l'apologétique patristique, mais de façon plus énergique et systématique : il s'agit de la constitution d'un " mythe chrétien ", qui aura la vie dure à travers tout l'âge classique. Nous voilà bien devant une reprise d'héritage qui est une création. La construction d'Annius a paru si grandiose aux hommes du $x^{e}{ }^{e}$ siècle que, même quand ils l'ont critiquée, ils ont admis beaucoup de ses présupposés. Le lecteur admettra facilement que le titre de la contribution d'A. Grafton ne soit pas qu'un brillant jeu de mots ( Invention of Traditions and Traditions of Invention in Renaissance Europe : The Strange Case of Annius of Viterbo "). L'invention de (fausses) traditions par Annius a laissé une trace historique profonde, sous forme d'une métamorphose en " traditions d'une invention » : cette coquille vide a été prise pour le fossile de quelque animal vivant.

Le succès éditorial du De Inventione Dialectica (au moins 70 éditions de 1515 à 1600), dû à la plume de Rudolph Agricola, et le long laps de temps (40 ans!) écoulé entre la rédaction du livre et sa première impression à Louvain font problème et offrent un autre lieu de réflexion sur le problème de la création culturelle. Les appréciations négatives portées sur cet ouvrage par les historiens de la logique tiennent à leur spécialisation. Ils n'ont pas vu que l'initiative éditoriale prise par le "cercle d'Erasme " constitue un effort pour donner à la culture de l'époque moderne un nouvelle forme de logique, une logique de l' « invention », substituée à la pureté immaculée, mais, pensent-ils, stérile, de la logique formelle. Cette erreur a déjà été mise à nu, des travaux de W. J. Ong à ceux de Lise Jardine (auteur de la contribution ici analysée). Mais Lise Jardine va maintenant plus loin. Elle estime que l'initiative du " cercle d'Erasme " a pour but de donner à l'humanisme flamand ses lettres de noblesse : la manière même dont est présentée la 
filiation des Italiens aux Flamands, par Erasme et ses amis, offre tous les traits d'un " conte de légitimation " ( qu'Agricola s'est déplacé pour s'instruire de sa Frise natale vers Ferrare.

Deux contributions (l'une relative à la conquête du Mexique par les Espagnols, l'autre à la vision occidentale du " despotisme oriental ») cherchent à méditer sur les difficultés de la véridique appréhension d'autrui. Pour la conquête du Mexique, Inga Clendinnen conteste la thèse de $T$. Todorov selon laquelle les succès de Cortès seraient dûs à sa remarquable capacité de manipulation des signes (du système culturel indien). Selon elle, au contraire, l'affrontement des Espagnols et des indigènes met en évidence le face à face de deux systèmes culturels, qui se révèlent de plus en plus étrangers l'un à l'autre, y compris dans les us et coutumes de la guerre : chacun ne peut déchiffrer les signes émis par l'autre que dans son propre code de la guerre ou de la paix, de la domination ou de la soumission. Pour « le despotisme oriental », Lucette Valensi soutient que la progressive dépréciation des institutions ottomanes dans les rapports des ambassadeurs de Venise, à partir de la fin du xvi siècle, s'explique plus par la modification du regard de l'observateur que par celle de la réalité observée. L'importance croissante accordée par les Vénitiens aux libertés de leur République fait surgir par contraste le monstre du "despotisme oriental ».

Le texte de D. R. Kelley (auteur déjà connu par de remarquables recherches sur les liens entre le droit et la naissance de l'histoire, notamment Foundations of Modern Historical Scholarship, Columbia University Press, 1970), montre de façon très documentée que le travail sur le droit coutumier accompli en Europe, à partir du Moyen Âge a produit une attention spéciale à la particularité des sociétés humaines et à leurs conditionnements géohistoriques: l'histoire du droit constitue donc la source de la science des sociétés, nouvelle preuve que l'innovation culturelle s'effectue souvent dans et par l'attachement aux traditions (les coutumes locales étant partout opposées à l'oppression «savante " du droit romain).

La contribution d'Alan Charles Kors vient renforcer une conviction de plus en plus fréquente chez les historiens: la pensée des Lumières n'est pas interprétée correctement si l'on n'y voit qu'un propos d'opposition au christianisme. Elle est bien plutôt le pur produit des débats internes à la pensée chrétienne. La constatation avait déjà été faite pour expliquer le succès de la religion, du droit, de la morale naturels, à partir des années 1670 (travaux de Jacques Solé, de H. G. Reventlow, sur lesquels je me suis appuyé pour tirer les conclusions de L'Écriture, le sacré et l'histoire, Amsterdam/Maarsen, A.P.A.-Holland University-Press, 1986). Ici, un nouveau pas est fait, puisque A. C. Kors démontre que les débats induits par la philosophie cartésienne sur les preuves de l'existence de Dieu ont contribué à ruiner le premier moment de l'apologétique chrétienne (la démonstration rationnelle des " vérités de la théologie naturelle ») et ont rendu possible un athéisme auparavant impensable : pour devenir athée, le curé Meslier pouvait se contenter de lire les manuels de théologie de son temps. Lamennais et ses disciples, précédés par l'abbé Bergier, ont d'ailleurs tiré de ce fait les conclusions qui s'imposaient.

Deux contributions s'occupent de l'espace anglais. L'une montre comment la diffusion de l'idéal de chevalerie et l'assimilation de l'Angleterre au peuple élu dans le sermon puritain ont préparé en sous-œuvre la Révolution anglaise. Les 
jeunes gens arrivés à Londres de la campagne anglaise pour devenir d'honorables bourgeois se percevaient imaginairement comme les héros du nouvel Israël qui mettrait fin pour toujours à la trahison papiste (entendez la politique neutraliste de Jacques $I^{\text {er }}$ dans la guerre de Trente Ans et le philocatholicisme de Charles $I^{\text {er }}$ ). L'autre contribution analyse à propos des poursuites judiciaires pour adultère (en Angleterre de 1670 à 1857) les interactions du droit et des intérêts économiques avec les conceptions de l'honneur masculin et du rôle féminin dans la famille et le couple. Non seulement les mœurs évoluent sous l'apparente immobilité des pratiques juridiques, mais la publicité faite aux procès pour cause d'adultère divulgue des récits scandaleux et contribue au changement de la loi.

À travers les méandres de ce compte rendu, rendus inévitables par la diversité des sujets traités, l'on pourra tout de même mesurer la fécondité des méthodes d'histoire culturelle mises à l'épreuve par le Davis Center de Princeton, sous l'impulsion d'Anthony Grafton. Entre le privilège accordé par l'histoire des mentalités à la très longue durée des systèmes symboliques et l'attirance exclusive pour les « ruptures épistémologiques " (l'un des auteurs parle, non sans ironie, de la " parthénogenèse " de l'innovation culturelle), l'historiographie américaine poursuit tranquillement, intelligemment, l'exploration d'un domaine qui la fascine, puisqu'elle y cherche inlassablement ses propres origines intellectuelles.

François LAPLANCHE.

New Perspectives on Renaissance Thougt. Essays in the History of Science. Education and Philosophy in Memory of Charles B. Schmitt. Ed. by John HENRY and Sarah Hurron. Londres, Duckworth \& Istituto Italiano per gli Studi Filosofici, 1990. $16 \times 24,324$ p., bibliogr., index.

Il s'agit d'un volume composé en mémoire de Charles B. Schmitt (1933-1986), prématurément disparu, dont un excellent texte de Luce Giard retrace la carrière et présente les orientations de recherche (p. 264-290). L'article est complété par une bibliographie des écrits de Schmitt due à Constance T. Blackwell (p. 291-308). Luce Giard montre comment, sur les traces de son maitre P. O. Kristeller, Charles B. Schmitt, avec beaucoup de sérieux et de modestie scientifique, a renouvelé notre connaissance de la culture du $\mathrm{xvi}^{e}$ siècle, en insistant plutôt sur les transformations de l'aristotélisme médiéval que sur l'opposition un peu factice entre platonisme et aristotélisme. C. B. Schmitt a aussi mis en lumière l'inadéquation des divisions actuelles du champ scientifique pour une exacte intelligence du travail de la pensée européenne au $x v^{e}$ siècle. Ce renouvellement de la vision résulte de deux efforts : l'un, pour prendre connaissance des immenses réserves de textes manuscrits conservés en Italie du Nord; l'autre, pour se rendre réellement contemporain des représentations et des procédures des hommes du $\mathbf{x v I}^{e}$ siècle. Ces efforts ont abouti aux magnifiques instruments de travail que sont $A$ Critical Survey and Bibliography of Studies on Renaissance Aristotelianism 1958-1969, Padoue, 1971 et The Cambridge History of Renaissance Philosophy, Cambridge University Press, 1988. 
En hommage à Schmitt, les auteurs du recueil ont cherché à avancer dans les directions majeures ouvertes par ses travaux. Sans prétendre à l'exhaustivité, nous chercherons à indiquer ce qui nous a paru s'inscrire le mieux dans les efforts actuellement accomplis pour renouveler l'histoire culturelle de l'Europe moderne (il ne sera pas inutile de rapprocher ce compte rendu de celui qui a été consacré au volume édité par A. T. Grafton et A. Blair, The Transmission of Culture in Early Modern Europ (voir supra p. 551-554).

1) Luce Giard signale que Schmitt s'est peu intéressé aux thèmes de la pensée religieuse. Cette réserve est abandonnée dans les communications de $R$. Popkin et de L. W. B. Brockliss. Le premier vient apporter une nouvelle pierre à la démonstration déjà accomplie sur d'autres bases, que l'incroyance des Lumières est un " effet pervers" des controverses religieuses des $x^{e}{ }^{e}$ et $x v I^{e}$ siècles (il s'agit ici de la polémique antichrétienne des écrivains juifs). L. W. B. Brockliss, dans une étude très neuve, estime que, dans le refus opposé par les théologiens au système héliocentrique, le poids de la lettre scripturaire a moins joué que l'attachement à l'autorité pontificale, mise en cause par les jansénistes dans les questions de fait, et défendue, en revanche, par les jésuites.

2) Les communications de M. J. B. Allen et de Dilwyn Knox s'interrogent sur les rapports entre philosophe et poésie (à propos du néoplatonisme de Marsile Ficin); entre langage et geste (à propos de l'intérêt porté à la symbolique gestuelle par Arias Montano et d'autres auteurs, jusqu'à $F$. Bacon, inclusivement) : la valeur universelle de communication inhérente à cette symbolique oriente les esprits vers d'autres signifiants que le mot, par exemple vers le hiéroglyphe. À travers ces recherches, se lit une certaine nostalgie de l'origine qui, pour « mythique » qu'elle soit, valorise d'autres formes d'expression que le discours cher à la logique, la grammaire et la rhétorique.

3) Le rapport du particulier et de l'universel dans la culture est abordé aussi dans la contribution de Donald R. Kelley sur la " coutume " (au sens juridique du terme). Celle-ci se légitime à la fois par sa durée dans le temps et son enracinement dans un espace : toute réflexion sur la valeur et la fonction de la coutume implique donc une prise de position anthropologique, qui contrebalance le monopole idéologique que le droit naturel acquiert à l'époque moderne. Malgré l'opposition de la Révolution française à la prise en compte des coutumes et sa préférence affichée pour le " droit de nature ", le xix ${ }^{e}$ siècle s'intéressera de nouveau à l'enseignement anthropologique véhiculé par la reconnaissance des différences culturelles.

4) Quatre communications sont consacrées à l'aristotélisme du $x^{i}{ }^{e}$ siècle. Dans la mesure où son enseignement est assuré par des chrétiens, s'accuse la tendance à séparer la métaphysique (pensée maintenant comme une propédeutique à la théologie chrétienne) et la physique (l'aristotélisme reste intéressant pour son côté empirique). Cette séparation permet à l'aristotélisme, en tant que cadre général de la pensée scientifique, de devenir éclectique et d'absorber des vues nouvelles, comme le magnétisme de William Gilbert.

5) D'autres communications montrent l'interaction constante à l'époque moderne entre les préoccupations politiques et les développements de l'érudition (par exemple, l'étude de l'histoire romaine sert à justifier la colonisation de l'Irlande), le rôle des pouvoirs dans le développement universitaire, l'importance 
du marché du livre pour la circulation des idées nouvelles, la singularité de la position occupée par la médecine, écartelée par sa double appartenance à la physique et aux arts.

Ce livre d'hommage à C. B. Schmitt ne dispense pas de lire Kristeller et Schmitt eux-mêmes. Mais la richesse de l'éventail déployé manifeste combien ils ont été de grands remueurs d'idées et d'intelligents éveilleurs de vocations érudites : de leur ceuvre, l'histoire culturelle de la Renaissance est sortie profondément transformée.

François LAPLANCHE.

Jean Bernhardt, Hobbes. Paris, Presses universitaires de France, 1989. 11,3 $\times$ 17,5, 126 p. ("Que sais-je?", 2498).

Thomas Hobbes. Philosophie première, théorie de la science et politique. Sous la dir. de Yves-Charles ZaRKA, avec la collab. de Jean BERnhardT, introd. de Raymond Polin. Paris, Presses universitaires de France, 1990. 17,5 × 24, 418 p. (« Léviathan »).

Les deux décennies qui viennent de s'écouler ont connu un grand nombre de publications sur Hobbes et sa philosophie, et il convient de saluer la production française même si elle est peu citée dans les bibliographies anglo-saxonnes, les Français étant loin d'être les seuls à ne pas lire les langues étrangères.

Enfin! Hobbes fait son entrée dans la collection "Que sais-je? ?. Le but du livre est d'orienter juristes, historiens du droit et amateurs de philosophie politique vers les données historiques et biographiques et vers les analyses qui permettront de mieux situer les idées politiques d'un penseur recouvert de mythes, caractérisé par une audace radicale et une véracité sans détour.

Suivant une courte introduction, le premier chapitre (22 pages, dont 13 consacrées au Short Tract on First Principles) met en évidence l'enseignement aristotélicien, l'idéal de rigueur, de clarté, d'universalité, et, avec la certitude dogmatique, l'organisation systématique du savoir. J. Bernhardt souligne qu'il n'y a pas de preuve que Hobbes ait pu être influencé, lors de son premier voyage en Italie, par les Padouans et par Galilée; il décrit avec prudence les relations Hobbes-Bacon, explique la découverte tardive d'Euclide, reprend ses propres thèses sur la relation Hobbes-Thucydide. De longues pages sont consacrées au Court traité, à la richesse et à la nouveauté des conceptions, au style, à l'influence de Suarez, aux thèmes abordés (théorie de la lumière et de la vision, conception mécaniste du monde, sensations, images), au caractère moderne malgré certainęs survivances scolastiques (magnétisme), à l'ambiance puritaine à l'université d'Oxford et au modernisme de Bacon.

La plus grande partie (49 pages, $2 / 5$ du livre) est le second chapitre qui consacre 29 pages aux Elements of Law. Dans une reconstitution vivante, où Mersenne, Galilée, Descartes sont bien au rendez-vous de la pensée - qui, à la faveur de la rencontre, de l'échange, de la dispute, se construit - , on voit le philosophe 
se mettre « en quête des principes de la science de la nature » et naître l'idée d'un grand système des sciences, d'un projet encyclopédique. L'étude du langage, de la théorie optique, du mouvement vital montre la mise en place de la réflexion anthropologico-politique, de la synthèse entre la tradition sophistique des "lumières grecques" et un rationalisme de l'ordre, entre la culture littéraire, l'expérience politique et la pensée de l'homme de science.

Jean Bernhardt parle des années d'exil et des années de l'exil. Ce n'est peutêtre pas un hasard si, pour écrire son autobiographie en vers, Hobbes s'est inspiré d'Ovide. Descartes aussi s'en fut ailleurs, où il déménagea souvent. L'exil fut propice à l'œuvre : au De Cive, avec le parfum de scandale que dégageait la synthèse hobbesienne (c'est l'adjectif désormais consacré) de l'individualisme et de l'autoritarisme, et au Léviathan (14 pages lui sont consacrées) où Jean Bernhardt, à contre-courant d'une longue tradition, voit Hobbes professer un christianisme rationaliste original de la tendance du puritain William Ames. La science doit être mise en complémentarité avec la foi et la tradition historique chrétienne. Même l'argument de cohérence ne rendrait pas illégitime chez le lecteur une conviction disons plus inquiète. Convaincantes sans réticence sont en revanche les lignes sur la personnalité représentative. Les pages sur le De Corpore éclairent le lecteur sur l'argumentation de Jean Bernhardt pour qui Hobbes fut un " penseur trop souvent réduit à la science politique » alors que «la science de la paix civile ne pouvait être, à ses yeux, qu'un secteur au plus privilégié, mais en tout état de cause justiciable des mêmes principes théoriques généraux ». On a compris que ce petit livre ne manque pas son but : juristes, historiens du droit, anglicistes, amateurs de philosophie politique en seront plus éclairés.

Publié sous la responsabilité de J. Bernhardt et Y.-Ch. Zarka, Thomas Hobbes. Philosophie première, théorie de la science et politique est un gros livre qui réunit les Actes d'« un grand et beau colloque " (J. d'Hondt), tenu à Paris en 1988, et qui lui aussi participait de la résurrection, de la redécouverte, de la renaissance (R. Polin), de la pensée hobbesienne, actes publiés à la mémoire du professeur A. Pacchi, dans une collection récente, solide et prometteuse. L'ouvrage se signale par le nombre (29) des signatures, la collaboration C.N.R.S.-Universités, le caractère international, l'apparition d'une jeune génération de chercheurs.

Un volume de cette importance n'appelle que peu de remarques formelles : on regrettera «le protagoniste principal » (p. 340), « sépulchre » (p. 144), «fut» pour " fût " (p. 145), " où " pour « ou " (p. 255), " quoique " pour " quoi que " et « quelque " pour « quel que " (p. 236, 237), « lui » au lieu de « eux » (p. 372), "décades " pour "décennies" (p. 291), «se contiennent l'une à l'autre " (p. 271); on souhaiterait parfois que les références fussent mises à jour (p. 119 et suiv., 347 et suiv., 398 et suiv.), que les sources fussent citees (p. 170-171, 207), que les traductions fussent moins parsemées d'anglicismes (p. 176 et suiv., 211$212,254,255)$. Ce sont là des défauts mineurs.

La première partie ( 7 articles) est consacrée à la philosophie première dont Y.-Ch. Zarka souligne le " caractère transrégional dans l'organisation du savoir ". On passe d'une métaphysique de l'être à une philosophie première du corps. Ce qui pose la question fondamentale de la représentation. L'annihilation du réel 
permet au mécanisme de fonder à la rigueur la représentation dans une stricte correspondance à son objet (P. Magnard). En contrepoint de quoi, ou presque, J. Bernhardt met en garde contre la tendance excessive à se détourner des aspects semi-réalistes de la pensée de Hobbes. De la même rigoureuse façon, M. Pécharman, dans une étude sur cause, effet et action, fait remarquer que la philosophia prima n'atteste pas tout à fait de sa propre cohérence sur la question de la causalité, et invite à réfléchir sur l'imagination. B. Willms pense, lui, que c'est la politique qui est la philosophie première, que Léviathan est le fruit de la dissolution radicale qui marque le xvir siècle. Faut-il pour autant, rapprocher dissolution et post-modernisme, Hobbes et Lyotard ? C'est Léviathan au risque de l'histoire des idées, thème connu. Nous sommes en transition, de la philosophie proprement dite à l'histoire de la philosophie avec l'étude de Guenancia sur Hobbes et Descartes à propos de la question de la "chose ", et à l'histoire de la science avec l'article de A. Beaulieu sur Hobbes et Mersenne.

La théorie de la science est traitée dans 9 articles. C'est un objet difficile mais fécond quand il est abordé de front (J. Barnouw, A. Robinet, M. Blay). Il est plus négociable dans des études comparatives (Hobbes-Leibniz, Hobbes-Gassendi), lorsqu'il soulève des questions connexes (problèmes de langage, d'influence, de politique, de méthode). Ce qui prouve bien que ce livre a raison de vouloir restituer un Hobbes total, fils de son temps. Ces études sont enrichissantes aussi pour la réflexion sur le signe, la causalité historique, les questions lexicographiques, la problématique de l'adaptation audacieuse, pédagogique et métaphorique d'une démarche de linguistique contemporaine à un problème éthique et politique du grand siècle, le rôle du souverain philosophe, arbitre et protecteur de la communauté scientifique (on regrette l'absence de toute référence chez Foisneau à New Atlantis), l'artificialisme et le naturalisme, et les questions de réception d'une œuvre de philosophie dans et par son milieu et son temps.

La troisième partie s'intitule "Politique" et regroupe 12 articles. Cette progression quantitative n'est pas surprenante, car le nom de Hobbes, après tout, ne nous est pas parvenu à cause de la science ou de la philosophie première. On retrouve au cœur de ces articles les grands noms (Machiavel, Gassendi, Hegel), les grands thèmes (conceptions de la loi, de la souveraineté, de l'obéissance), les questions de contexte historique (Bacon, Jacques $1^{\text {ex }}$, Selden), des questions déjà rencontrées dans le cadre de l'épistémologie (conatus, représentation), et l'on aborde les questions de théologie. Les études de Lazerri (qui met à jour les différences entre les problématiques et donc les méthodes de Machiavel et Hobbes), de Rudolph, sur l'obligation et la vertu, dégagent les aspects anthropologiques, psychologiques et sociologiques des fondements de l'éthique. P. Carrive, O. Bloch, R. J. Ribeiro proposent des analyses appuyées sur le contexte historique qui sont stimulantes pour les substantiels rapports qu'elles établissent, les questions qu'elles posent au lecteur, les aperçus nouveaux ou les formulations neuves et convaincantes qu'elles proposent. C'est la vertu de la pensée vivante de savoir redire sans répéter : ainsi F. Tricaud raconte-t-il Léviathan comme un mythos et un Bildungsroman, ainsi S. Goyard-Fabre montre-t-elle que l'obéissance à la loi civile fait la citoyenneté en répondant à la métamorphose du droit de nature, qui est a-juridique, en un droit civil, qui est un droit public, ainsi R. Polin voit-il Hobbes retrouver les conclusions d'Aristote, dont il s'est déclaré l'ennemi juré. 
F. Lessay soumet l'obéissance au consentement du sujet à l'action du souverain et non seulement au souverain lui-même. P.-F. Moreau et A. Matheron consacrent leur lecture à Hobbes théologien, lecteur herméneute de l'Écriture et ils nous ramènent aux positions de J. Bernhardt dans son petit ouvrage. Érudition et rigueur sont au service de l'imagination, et contraignent à des remises en question ; la préhension de la vérité ne peut qu'y gagner. Il faut approfondir le champ, comme faisait Hegel, dont P. Garniron souligne la compréhension profonde et interprétative de la pensée politique de Hobbes. Passe un souffle d'altitude quand les grands lisent les grands et enseignent leur œuvre.

Publié dans d'excellents délais, ce livre riche et divers aurait été utilement complété d'un index des notions et d'un index des noms qui en eussent facilité la lecture à ceux, qu'il faut espérer nombreux, qui le liront et entreront ainsi dans l'espace de la pensée moderne qui détermine encore notre présent.

Louis Roux.

Arnold A. Rogow, Thomas Hobbes. Un radical au service de la Réaction. Trad. de l'anglais par Eddy TREVEs, avec la collab. de Jacques Brun. Paris, Presses universitaires de France, 1990. 15 × 21,8, 363 p. (" Question »).

Les traductions d'études étrangères sont rares; il convient donc de saluer cette biographie parue dans sa version originale en 1986. Elle contribue à enrichir le dossier hobbesien qui bénéficie aujourd'hui d'un intérêt éditorial renouvelé, puisque l'ensemble de l'œuvre est en cours de traduction et de publication, offrant au lecteur français l'éclectisme, la richesse et l'originalité d'une pensée trop souvent réduite à la seule figure du Léviathan, le «Dieu mortel » qui tient dans sa main droite le glaive, symbole de l'État et, dans sa main gauche, la crosse pastorale de la religion: «Hobbes lui-même et sa pensée, malheureusement pour ceux qui prônent la catégorisation, résistent à toute tentative de la comprendre dans une filiation, car toutes simplifient à l'extrême et déforment la réalité » (p. 300).

$\mathrm{Si}$ cette biographie accorde une importance que certains jugeront disproportionnée à l'anecdote - garder enfant une bougie allumée par crainte de l'obscurité éclaire-t-il d'une lueur inédite la nature de la pensée du Hobbes devenu adulte? (p. 293) -, on retiendra surtout l'abondant dossier que constitue l'analyse des conditions historiques, de l'environnement intellectuel qui permettent de comprendre la genèse d'une des œuvres les plus fortes et controversées de la pensée politique, jugée «monstrosissimus » (p. 268) par bien des contemporains qui y lirent avant tout une scandaleuse apologie de l'athéisme (Arnold Rogow rappelle que, selon une estimation précise, 371 pages du Léviathan - sur les 714 pages que compte l'ouvrage - traitent de la religion). Car la longue vie de Thomas Hobbes (1588-1679) s'identifie d'une certaine manière à quelques-unes des contradictions d'un temps que les historiens ont appelé le «sombre xviı siècle". Confronté à l" " âpreté de la vie ", témoin de nombre des conséquences ravageuses de la guerre de Trente ans, de la guerre civile en Angleterre, de la Fronde en 
France, échappant à bien des maladies et à plusieurs épidémies - dont la peste à Londres -, l'auteur (entre autres) du De Cive (1642) et du Léviathan (1651) participe aussi à la révolution scientifique qui marque la première moitié du siècle et fonde notre modernité : très intéressé par l'optique, il a pour ami Harvey, Mersenne, Gassendi. Même s'il ne partage pas ses conclusions, il n'ignore rien des œuvres de Descartes. Il découvre Euclide à Genève (1630) et avec lui la géométrie, qu'il croit capable de conférer un statut scientifique à la politique : " La confiance de Hobbes dans l'enseignement des mathématiques, comme source de vérité échappant à la controverse et à la discussion, doit certainement quelque chose à Galilée et à Descartes, tous deux mathématiciens, dont les écrits transformaient et mettaient au défi les idées traditionnelles sur la nature de l'homme et de l'histoire [...]. Sa quête personnelle d'ordre et de sécurité rejoignait la pensée politique et intellectuelle de son époque et témoignait de sa détermination d'élaborer une science fondèe sur des principes politiques qu'il chérissait, de prouver, comme l'écrit son ami Mersenne, que $\mathrm{De}$ Cive et ses divers autres ouvrages étaient porteurs d'une vérité en quelque sorte non moins rigoureuse que les Eléments d'Euclide " (p. 108). André Rogow insiste sur les interactions permanentes entre les nombreux épisodes de la vie d'un écrivain qui se dit persécuté - déboires multiples, controverses âpres (une "guerre de Trente ans intellectuelle " l'oppose à des professeurs d'Oxford puis à la Société royale), exils forcés - et son pessimisme absolu sur la nature de l'homme, fondement et matrice de son système politique dont les prémices apparaissent dès la première cuvre, la traduction de l'Histoire de la guerre du Péloponnèse de Thucydide (parue en 1629) : « L'intérêt principal de Thucydide, mis à part son désir de faire le récit de la guerre, était à la fois d'établir le rôle décisif de la nature humaine dans la formation et la chute des sociétés et des individus, de démontrer les effets corrupteurs de la guerre sur la morale et les comportements, et de poser la question de savoir si la liberté ou la démocratie, la discipline et la maîtrise de soi peuvent coexister » (p. 80).

Cette volonté de démontrer la relation indissociable entre la nature mauvaise de l'homme - « la vie à l'état de nature est solitaire, pauvre, désagréable, bestiale et brève " - et la forme d'autorité politique pensée comme nécessaire, régulatrice et absolue pour faire barrage aux " passions " et instaurer la paix (militaire ou civile) continue aujourd'hui comme hier à nous interroger. Le « Dieu mortel » de Thomas Hobbes est la plus fascinante et terrible invention des hommes contre la peur de la nuit. 
Sir Robert Filmer, Patriarcha ou le pouvoir naturel des rois, suivi des Observations sur Hobbes. Prés. de Patrick Thierry, trad. de Michaël Bızıou, Colas Duflo, Hélène Pharabod, Patrick Thierry, Béatrice Trotignon. Paris, École normale supérieure de Fontenay/Saint-Cloud/L'harmattan, 1991. 13;4 X 21,6, 205 p. (« Logiques sociales »).

Sir Robert Filmer, Patriarcha and Other Writings. Dir. Johann P. Sommerville. Cambridge, Cambridge University Press, 1991. $14 \times 21,5,327$ p., index.

La réputation de certains auteurs est attachée à l'attention privilégiée que leur ont accordée, dans des œuvres majeures, des penseurs importants. Ainsi Robert Filmer, pris à partie par Locke dans les Deux traités du gouvernement civil. Mais Patriarcha, cible explicite de Locke, a longtemps été boudé par les traducteurs. C'est dire tout l'intérêt que pouvait présenter la première traduction française d'un des ouvrages maudits parmi les plus connus de la philosophie politique moderne. Malheureusement, l'édition dirigée et présentée par Patrick Thierry, fruit d'un travail collectif mené par un groupe de traducteurs de l'École normale supérieure de Fontenay/Saint-Cloud, ne procure pas toutes les satisfactions qu'on était en droit d'attendre de pareille entreprise.

Patrick Thierry signe une " présentation » de Patriarcha qui s'efforce de réhabiliter l'écrivain absolutiste que fut Filmer. De nombreuses coquilles (on en dénombre près de soixante-dix sur les quelque soixante pages de la présentation, notes non comprises) en gênent parfois gravement la lecture, mais là n'est pas l'essentiel. Il semble qu'en réalité le texte de Filmer résiste à toute tentative sérieuse de réhabilitation. Les arguments principaux ne sont pas neufs. $P$. Thierry met en avant la force que donne à Filmer sa connaissance de l'histoire anglaise. On ne voit pas bien comment le suivre sur ce terrain. Certes, Filmer cite de bonnes sources. Mais tous ses textes, Patriarcha en tête, ont la faiblesse majeure d'osciller constamment entre fait et droit sans que l'on sache bien quelle est la position théorique de Filmer sur leurs rapports. Or les faits ne peuvent avoir valeur de preuve qu'à l'intérieur d'un système théorique explicite permettant de les interpréter, et la grille d'interprétation appliquée par Filmer n'est autre que l'idéologie absolutiste, c'est-à-dire avant tout un ensemble de postulats implicites qui ne dépassent pas le stade du préjugé. C'est ainsi que certains commentateurs anglais, par exemple John Dunn, ont analysé l'œuvre de Filmer'.

Cette position idéologique est connue : nature et création sont les maîtres mots sur lesquels Filmer brode son mythe de l'autorité royale, avec une déficience conceptuelle qui affaiblit considérablement ce que certains auteurs retiennent parfois de ses écrits, c'est-à-dire sa critique, réputée imparable, du contractualisme. Filmer, en effet, croit avoir réduit à néant les théories contractuelles en sommant leurs partisans de préciser où et quand ce fameux contrat a été consenti. Non seulement la nature philosophique du contrat lui échappe (il s'agit avant tout d'un concept), mais il oppose à ce qu'il croit être une thèse historique une autre thèse

5. John Dunn, La Pensée politique de John Locke, Paris, Presses universitaires de France, 1991. 
historique qui mobilise une lecture fondamentaliste de la Genèse. P. Thierry a beau préciser que ce type de lectures est fort en vogue en Angleterre dans la première moitié du xvi siècle, cela n'apporte aucune caution proprement intellectuelle à Filmer. Au demeurant, affirmer que "personne ne remet en question l'interprétation littérale de la Bible » (p. 39) est excessif et devient un anachronisme au moment où Locke rédige ses traités. L'important est que Filmer, sur ce point précis et en général, entretient encore une fois une confusion fâcheuse entre le fait et le droit, et que l'opportunisme idéologique semble seul dicter la forme que prennent ses arguments successifs.

Il est, du reste, très insuffisant, si l'on veut montrer la faiblesse des adversaires de Filmer, de s'en tenir à la seule réponse de Locke. Algernon Sidney, James Tyrrell et, plus tard, Rousseau ont écrit contre l'auteur de Patriarcha des pages dont il faut tenir compte avant de s'essayer à le réhabiliter. Quant à voir en lui « un assez bon historien au sens plus moderne du terme " (p. 40), cela s'apparente à de l'abus de langage. En matière d'utilisation idéologique de l'histoire, l'étude comparée des défauts respectifs de la démocratie et de la monarchie, dans Patriarcha, reste un morceau d'anthologie. Filmer met à contribution les auteurs les moins favorables au régime qu'il dénonce et réduit les effets de la démocratie à un tableau sanglant de la guerre civile emprunté à Plutarque (p. 127-128). Il est possible que ce genre de rhétorique ait pu emporter la conviction de contemporains déjà acquis à sa cause, mais il est difficile de croire que cela réponde aux exigences de la pratique moderne de l'histoire. Au reste, si l'on veut se faire une idée exacte de Filmer historien, ce n'est pas Patriarcha qu'il faut lire, mais, par exemple, et comme le rappelle à juste titre P. Thierry (p. 14), The Free-Holders Grand Inquest (1648) ou les Observations upon Aristotles Politiques (1652). Il semble que la révision du cas Filmer, à laquelle se livre sans succès P. Thierry, réponde avant tout à des exigences de mise en valeur de l'auteur publié, un peu comme si l'éditeur n'avait pas assez confiance dans l'intérêt intellectuel que peut éprouver le public concerné par cette publication. La valeur documentaire de ce texte est pourtant bien suffisante pour légitimer une traduction. En outre, n'est-il pas contradictoire de prétendre rendre Filmer intéressant pour lui-même et, simultanément, de traduire de lui un texte inachevé, dont l'établissement fait problème, et qui n'a de notoriété qu'en raison de ses enjeux historiques ? On en verra une preuve dans le fait que la présentation du texte s'éloigne parfois beaucoup de Filmer (p. 58-61 notamment) et, en revanche, parle beaucoup de Locke. De plus, si l'on tient absolument à retrouver la "fraîcheur intellectuelle " que procure Patriarcha sans ses commentaires, pourquoi alors choisir comme texte de travail, au lieu du dernier état manuscrit, une édition posthume, en s'autorisant paradoxalement du fait que c'est celle que Locke a lue ?

Quelques reproches doivent être adressés à la traduction elle-même, dont les imperfections sont trop nombreuses pour qu'on puisse les passer sous silence. Comme l'introduction, mais dans une moindre mesure, le texte de Filmer souffre de fautes de français dont on veut croire qu'elles sont dues à l'absence de relecture d'épreuves mal composées ${ }^{6}$. Plus graves sont les erreurs manifestes qui altèrent

6. La « coquille » la plus étonnante est “ Dieu » mis pour « roi », p. 145. Un quid a sauté de la citation latine tirée de Tertullien, p. 142. On déplore l'absence quasi systématique des 
parfois, sur une page ou deux, le propos de l'auteur. On retiendra essentiellement l'affaiblissement de l'ironie et de l'excès propres à Filmer, dans des passages cruciaux où ce dernier déverse sa bile sur le régime qu'il hait plus que tous les autres, la démocratie. Là où Filmer dit clairement que, « quand bien même ils seraient innombrables ", les inconvénients de la monarchie ne sauraient peser plus lourd que l'immense risque que fait courir la démocratie, à savoir la sédition, on lui fait dire (p. 127) que, "bien qu'ils n'aient jamais été nombreux ", etc., ce qui change tout. En illustrant « l'apogée de la liberté romaine " par le fait que « tout homme pouvait être tué, qui le désirait ", Filmer faisait preuve d'une ironie qui disparaît dès lors qu'on traduit, sans raison : «tout homme pouvait être tué par quiconque le voulait " (p. 127). De même l'extrait de Plutarque décrivant les ravages de la guerre civile (conséquence inévitable, pour Filmer, de la démocratie) est édulcoré : les hommes ne sont plus égorgés dans les bras de leurs femmes (ce qu'il faut lire) mais auprès d'elles, et Sylla donne simplement des épouses (et non plus les épouses d'autres hommes) en mariage à certains (p. 128).

Quant à la justification (p. 74) du choix de traduire "commonwealth " par " état », elle n'est pas convaincante. On voit mal pourquoi le choix de « république " ne convient pas. Il a pour lui la pratique la plus établie et aucun des arguments avancés à son encontre ne porte vraiment. S'il est exact que, comme le souligne P. Thierry, Filmer établit une distinction entre "commonwealth " et "commonweal " dans ses Observations sur Hobbes, en revanche aucune cohérence stricte n'est observable dans l'emploi des mêmes termes dans Patriarcha. Le seul moment (p. 112) où les traducteurs se soient crus obligés de préciser entre parenthèses le terme anglais qu'ils traduisent n'est guère probant. En effet, si l'édition Chiswell de 1680 , choisie pour la traduction, porte bien, dans le même paragraphe, les deux termes concurrents, il n'en va pas de même dans les manuscrits. En pareil cas, il n'est pas évident que l'édition retenue doive faire autorité, même si c'est celle que Locke a lue. En tout cas, la précision lexicale n'est pas, dans ce passage, porteuse d'un écart sémantique qui mérite d'être si nettement signalé?

En attendant la parution de la traduction de Patriarcha (et du Premier traité de Locke) que prépare Franck Lessay aux Presses universitaires de France, on pourra se reporter, pour une connaissance plus approfondie de Filmer, à Patriarcha and Other Writings, qui rassemble ses textes les plus importants dans une édition qui semble définitive. On regrettera toutefois l'absence du légendaire traité sur les sorcières, An Advertisement to the Jury-Men of England touching Witches (1653), d'autant plus intéressant qu'il permettrait d'approfondir la confrontation avec Bodin, grand inspirateur de l'absolutisme de Filmer et auteur, rappelons-le, d'une Démonomanie des sorciers (1580). D'autres textes rares de Filmer, sur le blas-

accents circonflexes, des traits d'union et des accents graves requis pour différencier " où » de " ou " et « là " de « la », ainsi que la récurrence de « si il » à la place de « s'il ». Précisons enfin que l'auteur de la théorie de l'« individualisme possessif » est C. B. Macpherson, et non « Mac Pherson » (p. 61, 72).

7. On s'étonne d'ailleurs qu'à la page suivante, Filmer utilisant en succession " commonwealth » et " commonweal ", les traducteurs, dérogeant à leur principe, aient traduit " état » dans les deux cas. 
phème (Of the Blasphemie against the Holy Ghost, 1647) et sur l'usure (Quaestio Quodlibetica; Or a Discourse whether it may be lawfull to take Use for Money, 1653) permettraient, quant à eux, de compléter le portrait d'un auteur connu surtout pour ses positions sur l'absolutisme, alors qu'il a pris parti sur toutes les questions qui préoccupèrent l'Angleterre intellectuelle du xvir siècle.

Jean-François BAILLON.

John Dunn, La Pensée politique de John Locke. Une présentation historique de la thèse exposée dans les Deux traités du gouvernement. Trad. de l'anglais par Jean-François Baillon. Paris, Presses universitaires de France, 1991. 17,5 $\times$ 24, 286 p., bibliogr., index (" Léviathan »).

L'ouvrage de John Dunn que vient de traduire Jean-François Bailion dans la collection " Léviathan " des Presses universitaires de France a paru en 1969. C'est dire qu'il était urgent que le public français puisse enfin juger sur pièces de la valeur et de la portée d'une interprétation de la pensée politique de Locke, devenue maintenant classique outre-Manche.

Au moment de la rédaction de l'ouvrage, les commentateurs de la pensée politique de Locke avaient le choix entre deux grandes interprétations de la doctrine exposée dans les Deux traités du gouvernement civil (1690). La première, que l'auteur qualifie de libérale ou de constitutionnaliste, mettait l'accent sur la théorie lockéenne du gouvernement représentatif. Elle voyait en Locke l'idéologue dévoué du parti "whig ", le père spirituel de la Glorieuse Révolution anglaise et, au-delà, l'inspirateur principal de la Constitution américaine. L'œuvre politique maîtresse de Locke était interprétée comme la fondation philosophique du droit constitutionnel libéral moderne ${ }^{8}$. La seconde interprétation que l'auteur qualifie de marxiste (quoiqu'elle soit aussi, à quelques nuances près, celle de Leo Strauss $^{9}$ !) mettait par contre l'accent sur la théorie lockéenne de la propriété privée et du travail et sur son lien avec la théorie de l'État tel qu'il apparaît par exemple dans cette formule du $\$ 124$ du Second traité : « La fin capitale et principale en vue de laquelle les hommes s'associent dans des républiques et se soumettent à des gouvernements, c'est la préservation de leur propriété. " Prolongeant certaines réflexions de Marx ${ }^{16}$, un auteur comme Macpherson croyait pouvoir trouver dans la théorie de Locke la justification idéologique de l'Etat de classe.

L'ouvrage de John Dunn se présente d'abord comme une réfutation de chacune de ces deux interprétations, plus exactement comme une réfutation de l'aptitude

8. Harold J. LASKI, The Rise of European Liberalism, Londres, 1962.

9. Leo Strauss, Natural Right and Histony, Chicago, 1953. C. B. Macpherson, The Political Theory of Possessiv Individualism, Oxford, 1962.

10. La pensée politique de Locke est " l'expression classique de l'idée que la société bourgeoise se fait du droit par opposition à la société féodale ", Karl Makx, Theories of Surplus Value, Moscou, s. d., I, p. 356 ; cité p. 208 , n. 4. 
de chacune de ces deux interprétations à rendre compte du sens que Locke luimême avait pu donner à sa doctrine. Si l'auteur concède que ces deux interprétations peuvent exprimer le sens que la doctrine politique de Locke peut revêtir à nos yeux, il nie qu'elles puissent rendre compte du sens que la doctrine de Locke avait pour son auteur. De là, le projet, énoncé dans le sous-titre de l'ouvrage, d'une " présentation historique " de la thèse centrale de Locke, autrement dit d'une présentation des raisons à la fois biographiques et doctrinales pour lesquelles celui-ci a élaboré la doctrine des Deux traités.

L'examen de la thèse constitutionnaliste occupe les trois premières parties de l'ouvrage. L'auteur lui-même qualifie d' "épineux et enchevêtré " le commentaire auquel il soumet les textes de Locke (p. 16) et l'on ne saurait sans doute mieux dire. Deux arguments principaux s'en dégagent cependant. Le premier est développé dans les chapitres consacrés au « développement intellectuel » de Locke et aux ouvrages publiés antérieurement aux Deux traités. Comme le rappelle J. Dunn, Locke n'a pas toujours été le libéral dont l'histoire a conservé le souvenir. Les premiers écrits politiques de Locke ${ }^{11}$ sont au contraire des écrits " conservateurs », développant une conception absolutiste du pouvoir politique légitime. Or la "conversion " libérale de Locke coïncide fâcheusement avec son entrée au service de Shaftesbury et avec la promotion sociale qui s'en est suivie. Voir dans le libéralisme de Locke l'effet de son adhésion aux thèses politiques du milieu dans lequel il allait désormais évoluer impliquerait donc nécessairement de lui prêter une sorte d'opportunisme idéologique. Or, comme le note J. Dunn, c'est là une conclusion qu'il est impossible de tirer : « ne faut-il voir dans le " libéralisme politique " de Locke que l'instrument contingent d'une mobilité sociale ascendante, ou bien faut-il voir dans ce qui finit par devenir une ascension sociale indubitable la conséquence " méritée " de son dévouement au "libéralisme politique " ? [...] C'est une question saisissante par sa brutalité [...] à l'heure actuelle il ne semble pas que nous en connaissions la réponse - il se peut d'ailleurs que nous ne la connaissions jamais » (p. 38). En réalité, nous le verrons, le dernier chapitre de l'ouvrage fournit une explication plausible de la "conversion " libérale de Locke et le libère ainsi en partie du soupçon d'opportunisme.

Le second argument opposé à la thèse constitutionnaliste occupe près de la moitié de l'ouvrage. L'auteur admet que les Deux traités de Locke sont bien une réponse au Patriarcha de Robert Filmer, dictée par la nécessité de fournir au parti whig une défense et illustration de ses thèses : "Quand le Patriarcha de Filmer fut enfin publié en 1680, il devint d'une nécessité urgente de fournir un contrepoids idéologique capable d'exposer les fondements de la doctrine exclusionniste de façon à l'intégrer solidement dans la continuité de l'ordre historique compact de la tradition gouvernementale anglaise et à le protéger des harcèlements railleurs du Patriarcha. Laslett a montré de manière très convaincante que le travail de Locke en vue des Deux traités remonte à la période de cette lutte politique " (p. 57) ${ }^{12}$. Le problème est que le Second traité contient beaucoup plus qu'il n'aurait été nécessaire à la seule défense et illustration de la cause whig : " quand

11. Two Tracts of Government, éd. Philipp Abams, Cambridge, 1967.

12. Two Treatises of Government, éd. Peter LASLETr, Cambridge, 1960. 
il en vient à écrire les Deux traités, la doctrine qui se dessine est notablement plus individualiste que ne peut l'expliquer la seule adhésion au programme politique de l'Exclusion " (p. 60). Les Whigs avaient surtout besoin d'une théorie du gouvernement représentatif qui justifiât le rôle qu'ils entendaient faire jouer au Parlement ainsi que d'une théorie claire de la prérogative. Si l'ouvrage de Locke répond bien à cette attente, il contient aussi toute une théorie de l'autonomie individuelle et de la subordination du pouvoir politique aux fins individuelles qui, s'il forme l'essentiel du libéralisme politique de Locke, est aussi bien étranger aux préoccupations des Whigs. D'où vient cet individualisme, cet attachement farouche et constamment réaffirmé à la primauté de l'individu sur l'État?

L'auteur était sur ce point contraint de se confronter à l'interprétation de Macpherson, puisque celle-ci repose justement sur une analyse du sens de l'individualisme lockéen. C'est la partie la plus intéressante et la plus convaincante de l'ouvrage. L'auteur développe une argumentation circonstanciée pour établir qu'en aucune façon on ne peut prêter à Locke l'intention d'avoir, d'une manière ou d'une autre, chercher à universaliser le mode d'être «bourgeois" pour en faire la racine sociale de son anthropologie. Il est difficile de restituer brièvement une argumentation riche et foisonnante mais nous retiendrons un argument qui constitue l'un des principaux apports de l'ouvrage et prépare l'interprétation finale. L'auteur montre en citant des textes manuscrits de Locke et des sermons de prédicateurs calvinistes contemporains de Locke, l'existence d'une adhésion doctrinale et personnelle de ce dernier à la théorie calviniste de la vocation. Sa vision du monde social était commandée par le principe selon lequel le travail est une obligation imposée aux hommes par Dieu, Dieu appelant chaque homme à une mission, une charge, une vocation particulière. Or, du point de vue de cette théorie de la vocation, "le service le plus humble que nous rendions dans une vocation honnête, même si ce n'est que labourer ou creuser, si nous le rendons dans l'obéissance et dans la conscience du Commandement de Dieu, est couronné par de larges récompenses ${ }^{13}$. Le « conservatisme » social de Locke, son peu de "goût " pour des idéaux de révolution sociale ne s'expliquerait donc nullement par son lien personnel avec " une classe dirigeante " (dont l'auteur montre que Locke est loin d'avoir eu une opinion flatteuse) mais par ses convictions religieuses.

Nous sommes ainsi conduits à la véritable thèse de l'ouvrage que l'auteur expose et argumente dans le dernier chapitre. L'interprétation développée par John Dunn revient en dernière analyse à soutenir que le libéralisme politique de Locke est la conséquence à la fois logique et psychologique de ses convictions religieuses. Locke aurait pensé ce qu'il a pensé non pas parce qu'il était l'agent dévoué du parti whig ou le porte-parole de la bourgeoisie capitaliste mais parce qu'il était un dévot calviniste qui aurait été conduit à soutenir des thèses libérales parce que seul un État de ce type pouvait permettre aux individus de s'acquitter de leurs devoirs envers Dieu. « En présentant les choses de façon schématique, la théorie sociale et politique de Locke doit être envisagée comme la constitution de valeurs sociales calvinistes, en l'absence d'une source terrestre d'autorité théolo-

13. Joseph HaLl, Works, Londres, 1628 ; cité p. 228 , n. 4. 
gique et en réponse à une série de défis particuliers " (p. 260). C'est la théologie de Locke qui fournit la clef de sa politique et c'est la biographie et la psychologie de Locke qui expliquent ses convictions religieuses. S’il se convertit au libéralisme dans les années 1665 , c'est parce qu'il réalise que la loi divine peut et doit être connue directement par les individus, qui en sont comptables devant Dieu. S'il « a besoin de la liberté de pensée " dans La Lettre sur la tolérance de 1667, "c'est parce qu'elle est une condition nécessaire de la vérité religieuse " (p. 265). Plus généralement, la limitation de la compétence légitime de l'État, qui forme l'essence du libéralisme, s'explique chez Locke par la nécessité de reconnaitre une sphère d'autonomie individuelle dans laquelle chaque homme, dans la manière dont il " entend " et pratique sa vocation, décide de son propre salut. Ce n'est pas pour permettre au chevalier d'industrie d'accroître sa puissance et sa jouissance que l'État doit voir son pouvoir limité ; c'est pour permettre aux individus de faire leur salut.

Il n'est pas besoin d'insister sur l'originalité et l'intérêt de cette interprétation de la pensée politique de Locke. Non seulement elle nous révèle un aspect méconnu de la personnalité intellectuelle de Locke mais surtout elle permet de poser, à propos du libéralisme politique dont il est effectivement le premier théoricien conséquent, un problème particulièrement décisif : le libéralisme politique peut-il être représenté comme une figure du Souverain Bien politique si on le sépare des prémisses religieuses qui le faisaient paraître tel à son inventeur? Le libéralisme politique est-il théoriquement et pratiquement viable si à l'anthropologie religieuse de Locke qui le premier l'avait conçue, on substitue - comme l'histoire ultérieure du libéralisme semble l'avoir fait - l'anthropologie hédoniste et athée de Hobbes? Si l'ouvrage de J. Dunn ne répond évidemment pas à cette question, il a au moins l'insigne mérite de conduire son lecteur à se la poser.

Stéphane Chauvier.

Les Universités européennes du xvf au xvIIf siècle : histoire des populations étudiantes. T. 2 : France. Dir. Dominique Julia, Jacques Revel. Paris, Ed. de l'École des hautes études en sciences sociales, 1989. $15 \times 23,616$ p., graph., tabl., cartes (« Recherches d'histoire et de sciences sociales », 18).

Cet ouvrage est le second tome d'un travail collectif consacré aux populations étudiantes européennes de l'époque moderne. Le premier tome portait sur les universités européennes à l'exception de la France; celui-ci étudie les étudiants en France. Il comporte deux types de contributions très différentes. Le premier regroupe quatre textes portant sur des points particuliers. J. Verger rappelle les difficultés spécifiques d'une histoire quantitative des universités médiévales, en particulier si l'on veut inclure une dimension prosopographique étendue. L. W. Brockliss propose deux contributions. Dans la première, il étudie les modèles de fréquentation scolaire à l'université de Paris en 1400 et 1800 d'où il conclut à leur stabilité durant ces quatre siècles. La seconde est consacrée à la for- 
mation du clergé britannique catholique à l'université de Paris entre 1426 et 1789 . P. Ferté, enfin, s'adonne à la description de la population étudiante du Rouergue au $x$ viri siècle. L'originalité de son travail vient surtout du fait qu'il ne s'agit pas d'étudier la population étudiante d'une université mais d'analyser en amont l'ensemble du contingent de départ d'une région délimitée.

Le second type est constitué par un gros article de portée générale signé de D. Julia et J. Revel et consacré aux étudiants et à leurs études dans l'ensemble des sites universitaires français. Le premier chapitre étudie les pérégrinations académiques. Dans l'ensemble des étudiants, « les pérégrinations ne constituent qu'une mince frange, une élite de la culture et de la fortune ". Mais au souci de connaître l'Europe des grandes universités s'ajoute souvent une raison moins avouable qui est la recherche d'un titre au moindre coût intellectuel et financier, motivation qui tend à devenir dominante dans le voyage estudiantin. La nouveauté majeure de l'Ancien Régime est la réorganisation progressive de l'espace universitaire aux $\mathrm{XVII}^{e}$ et XVIII ${ }^{e}$ siècles. Il tend à se rétrécir car chaque université s'efforce d'assurer l'ensemble de la formation des étudiants. Les deux auteurs insistent sur deux raisons complémentaires. La première est l'éclatement de l'ancienne chrétienté médiévale en confessions antagonistes qui oblige à redéfinir les itinéraires possibles. De nouveaux circuits apparaissent tel celui des étudiants partis vers les universités réformées étrangères comme Genève, Leyde, Bâle et Heidelberg qui constituent un véritable réseau réformé que les auteurs étudient en détail. La seconde raison est l'influence croissante des États modernes qui entendent peser plus autoritairement sur les universités pour contrôler le recrutement et la formation de leur bureaucratie, ce qui conduit progressivement à une nationalisation des grades. L'article se poursuit par l'étude systématique et successive des étudiants en droit, en théologie et en médecine. Les facultés de droit du $x^{e}{ }^{e}$ au xviI' siècle sont le lieu d'une opposition tenace entre la monarchie qui veut en faire un lieu d'apprentissage effectif pour former des officiers compétents et la résistance victorieuse de la société civile, surtout le monde des officiers qui veulent transmettre leur charge à leurs descendants. Les insuffisances de l'université qui en résultent déportent la formation juridique soit en amont du côté des familles, soit en aval parmi les compagnies d'officiers ou dans l'étude d'un procureur. C'est alors moins un savoir qu'une pratique qui se trouve valorisé. Le pouvoir royal tente de réformer les études juridiques pour empêcher la fraude aux dispositifs complexes et variés et restreindre le nombre d'étudiants de basse condition, improductifs et inutiles à l'État. C'est l'objectif de l'édit de Colbert de 1679, inefficace cependant dès sa proclamation car toute réforme est condamnée d'avance par le système de transmission des offices, âprement défendu par les grandes familles parlementaires. Plus tard, la réforme Maupeou qui veut introduire la méritocratie échoue également; c'est que, comme le montrent fort bien D. Julia et J. Revel, le grade n'est pas destiné à certifier un niveau de compétence mais à accéder à un corps professionnel.

Les études de théologie présentent un profil différent. D'abord, l'enseignement se trouve éclaté en plusieurs centres. Dès le $x^{e}{ }^{e}$ siècle, en effet, les collèges deviennent des centres d'enseignement autonomes et assurent une formation théologique qui concurrence celle des universités. Leur mouvement d'agrégation à ces dernières, voulue par la monarchie pour mieux les contrôler à une période de 
querelles gallicanes, permet de donner une assise juridique à cet enseignement. À la fin du Xvi ${ }^{e}$ siècle, les séminaires s'ouvrent à l'enseignement de la théologie et en constituent le troisième pôle. Dès lors, le conflit est ouvert au xvir ${ }^{e}$ siècle entre l'épiscopat et les universités qui voient leur fréquentation étudiante de plus en plus menacée. Les auteurs étudient ensuite le marché de l'emploi pour les théologiens et évaluent la possibilité pour les gradués de se voir attribuer des cures. La description des cursus qui clôt ce chapitre permet de souligner la spécificité des études de théologie où le mérite, à côté des privilèges de la naissance, a sa place en particulier à travers l'ordre de classement à la licence dont s'inquiètent les prélats.

Les études médicales sont particulières car ce sont celles qui préparent le plus explicitement à une activité professionnelle. Il en résulte une normalisation des règles universitaires bien avant les réformes de 1696 et 1707 qui, comme celles de 1679 en droit, tentent de juguler la fraude dont le principe essentiel est de jouer sur la différence entre facultés d'études (Paris et Montpellier) et celles décernant les grades, beaucoup plus complaisantes et sans enseignement sérieux. Le cas limite est celui d'Orange où le doctorat s'obtient avec une grande indulgence dans l'examen des connaissances et surtout à un coût de $60 \%$ inférieur à celui de Montpellier et 30 fois moindre qu'à Paris. Le réseau des facultés médicales est de ce fait fort disparate. Il est décrit en détail, cartes à l'appui, à partir de plusieurs critères : nombre des enseignants, des étudiants et nature des enseignements. Il en résulte une image bipolaire avec deux centres nationaux et un ensemble de facultés moyennes au rayonnement essentiellement régional.

Le chapitre final est consacré successivement à l'étude du recrutement géographique des étudiants, à leur origine sociale et aux conjonctures des universités, avec à chaque fois une distinction entre les trois facultés supérieures. L'impression qui prévaut est qu'entre le xvir et le xviri siècle, en médecine comme en droit surtout, le cas de la théologie est plus complexe, on tend à une normalisation de l'espace universitaire remodelé sur une base régionale mieux délimitée. Mais, dans les trois cas, s'affirme progressivement le rôle national des facultés parisiennes. Le repérage social est plus délicat parce qu'il nécessite une approche prosopographique. Un tableau statistique (p. 338) présente les résultats essentiels. Il montre que si le spectre social est relativement ouvert au xvi ${ }^{e}$ siècle, en particulier sur le monde de la boutique et de l'échoppe, voire des laboureurs, il se referme ultérieurement au profit d'une autoreproduction de chaque milieu professionnel. L'aspect patrimonial et quasi dynastique des professions médicales ne contredit pas cette analyse. L'enseignement de la théologie reste plus ouvert que celui du droit. Le souci monarchique, après 1650 et surtout 1740 , de contrôler la formation de l'élite ecclésiastique à l'université de Paris a favorisé le critère de l'excellence scolaire dont la communauté des robertins du séminaire Saint-Sulpice est le lieu d'élection. Le livre s'achève sur un retour au modèle de L. Stone, point de départ du premier tome. La confrontation suppose l'établissement d'un bilan global difficile à dresser à cause des divergences régionales et de disciplines. La thèse de L. Stone se trouve cependant démentie par la plupart des conjonctures étudiées qui ne confirment guère le schéma de la révolution éducative.

L'ouvrage impressionne par la densité de son contenu et la grande richesse de son information, sa forte annexe statistique en porte témoignage. Le titre retenu 
est réducteur car en plus d'une description quantitative des populations étudiantes et des conjonctures universitaires, ce sont tout à la fois les usages sociaux de l'enseignement, les stratégies éducatives, les transformations des cursus ou les liens avec la société dans son ensemble qui sont analysés. C'est peut-être l'articulation de cette diversité qui suscite le plus d'interrogations. En effet, un primat incontestable est accordé à l'approche quantifiée, inspiratrice d'une organisation interne du texte qui rend difficile toute lecture synthétique. On aurait aimé un traitement plus systématique, et pas seulement chiffré, de quelques-unes des problématiques suggérées, mais rarement centrales, ce qui aurait valorisé un texte dont la pertinence de nombre d'analyses est souvent remarquable et qui constitue désormais une référence essentielle pour l'histoire de l'éducation.

Jean-Yves GreniER.

L. W. B. BRockLIss, French Higher Education in the Seventeenth and Eighteenth Centuries. A Cultural History. Oxford, Clarendon Press, 1987. $16 \times 24$, xiii-544 p., bibliogr., index.

Le livre de L.W.B. Brockliss constitue une réalisation exemplaire des grandes monographies qu'il conviendrait de consacrer à l'histoire intellectuelle de l'Europe moderne. Le paradoxe est que cette réalisation (où il s'agit de la France) est due à une plume anglaise. L'historiographie française, il faut bien le dire, s'est trop longtemps détournée de ce type de travaux, le laissant aux Anglo-Saxons ou aux Italiens, comme si, dans notre pays, s'était instituée une division du travail entre les spécialistes des contenus (sciences, philosophie, droit, médecine, théologie) et les historiens des institutions (collèges, universités, académies, circuits du livre). La revue History of Universities, dont L. W. B. Brockliss a pris la direction, à la suite du regretté Charles B. Schmitt, refuse toute dichotomie et s'intéresse aussi bien au contenu qu'à l'organisation des savoirs : cette orientation commande aussi la rédaction de French Higher Education.

Ce gros livre de cinq cent quarante-quatre pages est constitué de quatre parties. La première "The Environment " est consacrée au personnel enseignant, à la vie étudiante, à la demande sociale de savoirs universitaires. Nous y apprenons que les universités françaises assurent avant tout, et cela jusqu'à la fin du xviII siècle, la formation de clercs et de juristes, la proportion d'étudiants en médecine restant faible. L'étude des implantations universitaires est conduite avec précision, accompagnée d'estimations touchant la population étudiante dans les différentes villes dotées d'une université.

La deuxième partie « The Propaedeutic Arts » insiste sur le rôle dominant du latin dans les humanités. Indispensable à cette époque même à l'étude des sciences, il connaît pourtant un certain recul dans la deuxième moitié du xvir' siècle; ce recul des "humanités" s'accentue encore dans le champ des études grecques. Les exercices scolaires se tournent, en effet, de plus en plus vers le perfectionnement de l'expression française. L'histoire est enseignée de manière 
plus utilitaire que critique (c'est-à-dire pour les exemples moraux qu'elle contient), la géographie s'enrichit, mais reste très descriptive (seule la fin du $\mathrm{xvIII}^{e}$ siècle voit s'ébaucher une géographie plus scientifique, situant le globe terreste dans le système solaire et utilisant les travaux de Buffon).

La troisième partie " The Ethical and Metaphysical Sciences » présente l'enseignement des sciences morales et philosophiques en soulignant la place prise par l'ontologie générale dans le cursus philosophique et par la critériologie de la connaissance historique dans le programme de la logique (ce qui confirme les grandes enquêtes de Paul Dibon: La Philosophie néerlandaise au siècle d'or, Paris/Amsterdam, 1954 et de Carlo Borghero : La Certezza e la storia, Milan, 1983). L'histoire de l'enseignement théologique en France aux xvit et xviI' siècles est excellemment dominée par L.W. B. Brockliss qui retrace à ce propos, avec beaucoup d'exactitude, la trame des grandes controverses religieuses qui secouèrent la France. Il ressort, de cette étude précise, des résultats qui remettent en cause d'abusives simplifications : ainsi le gallicanisme " massif " de la faculté de Théologie de Paris ou le naufrage de la morale jésuite dans le probabilisme. Ce grand chapitre de synthèse, aboutissant à des jugements équilibrés, mérite beaucoup d'éloges. L'auteur a bien vu aussi les déficits de l'enseignement théologique français au XviII siècle : abusant d'une méthode scolastique destinée à forger des convictions simples et solides chez les clercs, de plus en plus tourné vers l'apologétique, il est assez peu nourri d'exégèse et de science patristique.

La quatrième partie " The Natural Sciences " note le lent ajustement de l'aristotélisme à la nouvelle astronomie et à la nouvelle physique, dans la deuxième moitié du xviı siècle, puis le triomphe du cartésianisme en France et son recul, à partir de 1740, devant la conception newtonienne du monde. Les mathématiques prennent une place grandissante dans la formation des jeunes gens, mais elles s'enseignent souvent hors institution, chez des maîtres privés.

Cette importante enquête est complétée par une centaine de pages d'annexes, comprenant une prosopographie et une bibliographie très utiles. Mais l'intérêt du travail de L.W.B. Brockliss s'étend au-delà de ces aspects documentaires. La conclusion de l'ouvrage, très originale et très neuve, met en lumière le déséquilibre qui s'instaure, au temps des Lumières françaises, entre l'enseignement du mécanisme dans les collèges et le conservatisme de l'enseignement juridique et théologique, destiné à conforter l'ordre catholique et monarchique. L'enseignement scientifique, en diffusant les idées de "loi » et d' " utilité ", a été, selon L.W. B. Brockliss, le vecteur de la philosophie des Lumières. Cette percée de la science a fait davantage pour préparer la couche instruite du peuple français à accepter l'idéologie révolutionnaire, que la diffusion des nouvelles théories politiques et religieuses, qui n'étaient répandues dans les collèges qu'à des fins de réfutation. Comme, par ailleurs, le nombre de grands savants français ayant tenu une place dans l'enseignement reste fort petit au XVII ${ }^{e}$ siècle, il faut conclure que l'armée obscure des professeurs de collège a joué un rôle capital dans cette imprégnation scientifique et a préparé « l'avant-garde » de la Révolution. Cette conclusion, qui ne manque pas de hardiesse, montre la supériorité de l'étude systématique des pratiques culturelles sur l'impressionnisme de la traditionnelle « histoire des idées ", dès qu'il s'agit d'éclairer les causes d'une grande mutation intellec- 
tuelle : des idées, on peut indéfiniment discuter, tandis que les pratiques culturelles s'imposent.

François LAPLANCHE.

Otfried HöfF, La Justice politique. Fondement d'une philosophie critique du droit et de l'État. Trad. et adapté de l'allemand par Jean-Christophe Merle. Paris, Presses universitaires de France, 1991. 17,5 × 24, 397 p., bibliogr., index ( Léviathan »).

Le professeur Höffe s'est donné ici pour tâche, au nom d'une philosophie première, de légitimer le droit et la politique dans la perspective des modernes, non sans parfois tenir compte des anciens, à vrai dire dans une assez faible mesure à notre avis. S'inspirant librement de Rawls et de Kant, l'auteur cherche à montrer qu'au fondement du droit et de la politique se trouve la justice. Mais, afin de pouvoir s'opposer efficacement à l'utilitarisme, il ne peut s'agir que d'une justice distributivement comprise, c'est-à-dire mesurée par l'égalité fondamentale de chacun des individus qui composent le corps politique. Ceci permet d'échapper à l'idée d'une justice conçue comme bien matériel de la collectivité en tant que telle.

Höffe établit, à partir d'une analyse sémantique, la nécessité d'une telle justice, à l'encontre du positivisme, et plus spécialement du normativisme kelsenien, auquel il oppose principalement, outre la difficulté à rendre compte de certains phénomènes comme le droit coutumier, l'existence d'une liberté fondamentale, antérieure au droit et à l'État. Mais cette constatation risque de nous entraîner vers l'anarchisme, car, si nous sommes des sujets libres, il est nécessaire de légitimer le droit et l'État à partir de cette liberté et dans la perspective du développement de celle-ci. Cela disqualifie la pensée juridique classique, qui n'a pas suffisamment thématisé la légitimité de la contrainte, à cause d'un recours impatient à la nature et à une nature politique et coopératrice. Est également réfutée la prétention de la pensée classique à donner pour fin à la politique le bonheur. Dès lors, la protection et le développement de la liberté, qui sans cela serait menacée par toute autre liberté, fonde et légitime la constitution d'un État, dans la mesure où cette constitution, grâce à l'idée d'état de nature et de contrat social retrouvée dans une expérience de pensée, fait gagner à chacun de n'être plus victime de la liberté d'autrui. À partir de ces principes, l'auteur montre comment la fondation progressive de l'État se réfère à une anthropologie dont la liberté est la base, et dont les droits de l'homme sont l'expression. Il développe ensuite les grands traits de ce que pourrait être une politique de ce type.

Quelle que soit la pertinence de telle ou telle analyse particulière, la démarche de l'auteur ne parvient pas à nous convaincre. La discussion qu'il mène avec l'anarchisme le conduit à reprendre la vieille idée de l'état de nature, dont il estime qu'il nous met en présence d'un conflit fondamental entre des hommes brutalement « jetés-là». Le schéma du conflit prédomine donc à l'origine sur celui de la coopération, ce qui légitime à la fois la contrainte étatique et la réfuta- 
tion de la pensée classique. Or celle-ci se réfère à une réalité et à une nature plus fondamentales; elle s'appuie sur la constatation simple que nous sommes tous nés au sein d'un couple formé d'un homme et d'une femme après conception. Loin d'être "jetés-là », nous avons donc été formés dans la collaboration, et la naissance est la première manifestation de la nature. L'idée selon laquelle nous serions «jetés-là » n'est compréhensible que par référence aux mythes des commencements absolus. La réfutation de l'anarchisme passe donc beaucoup plus par son auto-disqualification qui résulte de son refus de prendre part à la communauté et au dialogue rationnel qui la caractérise.

L'allégeance de Höffe aux schémas de la pensée moderne ne lui permet pas non plus de s'opposer efficacement au positivisme, dans la mesure où il est amené à lui concéder que le droit se définit par la contrainte. En conséquence, il doit aussi reconnaître, et de fait reconnaît, la légitimité d'une "science du droit" neutre, à la manière kelsenienne. Il refuse aussi contre Radbruch, comme on s'y attendait, l'idée que les normes doivent être justes, non seulement dans leur fin générale, mais aussi dans leur particularité.

Enfin, l'individualisme foncier d'une telle pensée et la crainte de fournir des armes à l'utilitarisme l'obligent à négliger totalement la justice générale et l'effet éducatif de la loi, qui ne trouvent leur fondement que dans le développement d'une nature. La réponse de l'auteur à l'utilitarisme est donc fragile, car elle lui concède que la justice est un " gain ». Höffe appauvrit ainsi la notion de justice, comme en témoigne précisément son silence sur la justice générale.

On trouvera ici une très sérieuse tentative de rénovation des idées de l'école du droit naturel moderne, qui s'inspire d'un Hobbes tempéré par Kant, et de la remise à l'honneur de la justice par Rawls. Le choix qui nous est présenté est cependant trop limité. Il existe, dans la tradition de la philosophie politique, des pensées de la justice et de la nature, mieux fondées, nous semble-t-il. On voit mal, en effet, comment il serait possible de s'éloigner de la nature sans ouvrir la porte à la violence.

Michel BastTr. 